\title{
LRRK2 and the Stress Response: Interaction with MKKs and JNK-Interacting Proteins
}

\author{
$\begin{array}{lll}\text { C.H. Hsu } & \text { D. Chan } & \text { B. Wolozin } \\ & \text { a, b }\end{array}$ \\ Departments of a Pharmacology and ${ }^{b}$ Neurology, Boston University School of Medicine, Boston, Mass., USA
}

\section{Key Words}

MAP kinase $\cdot$ c-jun NH2-terminal kinase $\cdot$ Scaffold proteins $\cdot$

Parkinson's disease $\cdot$ Immunoprecipitation

\begin{abstract}
Increasing evidence supports a putative link between LRRK2 function and the MAP kinase cascades. We recently demonstrated that LRRK2 binds to MKK6, -3, and -7. Previous studies demonstrated that scaffold proteins are essential in the regulation of subcellular localization of stress kinase complexes. The c-jun NH2-terminal kinase (JNK)-interacting proteins (JIPs) are a group of scaffold proteins that play an important role in the regulation of MAP kinase signaling cascades. JIP1-3 are known to regulate the specificity and localization of the JNK pathway, while JIP4 is a specific scaffolding protein for the $\mathrm{p} 38$ pathway. We demonstrate that LRRK2 binds to JIP1-4, and is associated with increased levels of total JIP1, $-3,-4$, oligomeric JIP and ubiquitinated JIP. These results are consistent with a putative role of LRRK2 in regulating the stress kinase cascade.

Copyright $\odot 2010$ S. Karger AG, Basel
\end{abstract}

\section{Introduction}

Mutations in leucine-rich repeat kinase 2 (LRRK2) are a common cause of autosomal-dominant Parkinson's disease (PD) and also occur among some sporadic cases of PD $[1,2]$. The most prevalent mutation in European populations, G2019S, is associated with approximately $3 \%$ of sporadic PD and $30-40 \%$ of familial PD cases among Ashkenazi Jews and North African Arabs [3, 4]. Patients with LRRK2 mutation exhibit late disease onset with symptoms indistinguishable from idiopathic PD patients, and are also responsive to L-dopa treatment. The pathology associated with LRRK2 mutations is heterogeneous. Most cases of PD associated with mutations in LRRK2 display Lewy body pathology in the brainstem and substantia nigra - the most common pathological presentation of subjects with mutations in LRRK2 $[5,6]$. A small number of cases present with tau-positive neurofibrillary tangle pathology or with no visible inclusion pathology $[5,6]$.

The biological function of LRRK2 remains unclear. Greatest homology is seen between LRRK2 and the receptor interacting kinase (RIP) family of proteins, which regulate responses to death receptors $[7,8]$. In this context, it is notable that LRRK2 binds FADD, a death receptor-mediating protein [9]. LRRK2 is present in the cytoplasm but also associates with numerous membranous organelles, such as the mitochondria, endoplasmic reticulum, trans-Golgi, and plasma membrane [10]. LRRK2 contains several distinct functional domains. Three domains, COR, LRR and WD40, are proteinbinding domains. Most attention has focused on the ROC and MKKK domains, which exhibit enzymatic activity. The ROC domain binds GTP and exhibits low

\section{KARGER \\ Fax +4161306 1234 \\ E-Mail karger@karger.ch}

(C) 2010 S. Karger AG, Basel

www.karger.com
Accessible online at:

www.karger.com/ndd
Benjamin Wolozin

Departments of Pharmacology and Neurology, Boston University School of Medicine

72 East Concord St., R614

Boston, MA 02118-2526 (USA)

Tel. +1 617414 2652, Fax +1 617414 2651, E-Mail bwolozin@ bu.edu 
level GTPase activity $[11,12]$. Studies of LRRK2 ROC protein, produced as recombinant protein, demonstrate that the ROC domain has the capacity to dimerize [13]. Studies of LRRK2 using transfected cells also suggest that the full-length protein also dimerizes [14-17]. The MKKK domain shares homology with mixed lineage kinases, which regulate stress kinase cascades [18]. Multiple studies demonstrate that LRRK2 can autophosphorylate, although the amount of activity generated is low [19-21]. The G2019S LRRK2 mutation increases autophosphorylation activity [19-23]. Many other diseaselinked mutations also increase autophosphorylation, but the stimulation is less than for G2019S and does not occur with all disease-linked mutations. A number of putative targets of LRRK2 phosphorylation have been suggested, including E4-BP and moesin, but these results have yet to be confirmed by other groups [24,25].

Increasing evidence supports a putative link between LRRK2 function and the MAP kinase (MAPK) cascades. LRRK2 was recently shown to phosphorylate MKK3, -4, -6 and -7 [26]. We recently demonstrated that LRRK2 binds to MKK6, -3 , and -7 , and activates the $\mathrm{p} 38$ and c-jun $\mathrm{NH} 2$-terminal kinase (JNK) pathway [27]. Association of LRRK2 with MKKs stimulates expression and membrane translocation of these proteins, but does not strongly increase their phosphorylation. Localization of kinases is often facilitated by binding to scaffold proteins, which tether the kinases to particular membrane organelles and bring the kinases together with their substrates. Previous studies demonstrated that scaffold proteins are essential in the regulation of subcellular localization of stress kinase complexes. The JNK-interacting proteins (JIPs) are a group of scaffold proteins that play an important role in the regulation of MAPK signaling cascades [28]. JIP1-3 are known to regulate the specificity and localization of JNK pathway, while JIP4 is a specific scaffolding protein for the p38 pathway [28-30]. Given these data, we hypothesize that the JIP proteins would be integral for the regulation of LRRK2.

\section{Materials and Methods}

\section{Constructs}

Wild-type (WT) and mutant LRRK2 V5-His tagged and $2 \mathrm{xMyc}$ constructs were generated as described in Greggio et al. [20, 21]. Flag-tagged pcDNA3 JIP1-4 plasmids were generous gifts from Dr. Roger Davis (University of Massachusetts).
Cell Culture

HEK293-FT cells were maintained in DEM/10\% FBS. Transfections were performed using Lipofectamine 2000 (Invitrogen) as described previously [27, 31].

\section{Coimmunoprecipitation}

HEK293-FT cells were transfected with the appropriate constructs for $48 \mathrm{~h}$, and coimmunoprecipitations were performed as described previously $[27,32]$. Antibodies were purchased from Sigma (V5, Flag, c-myc, actin). Lysates and immunoprecipitates were loaded and resolved by 3-8\% NuPAGE Tris-acetate (for studies of JIPs) or 4-20\% Tris-glycine gels (for studies of MKK3) for Western blotting.

\section{Subcellular Fractionation}

HEK293-FT cells were transfected with the appropriate constructs for $48 \mathrm{~h}$, and subcellular fractionation was performed as described in Hsu et al. [27] to separate the samples into cytosolic and membrane fractions. Antibody against calnexin (Calbiochem) was used as the membrane marker, and antibody against GAPDH (Biodesign) was used as the cytosolic marker. Lysates were loaded and resolved by 3-8\% NuPAGE Tris-acetate (for studies of JIPs) or 4-20\% Tris-glycine gels (for studies of calnexin and GAPDH) for Western blotting.

\section{Statistical Analysis}

All data were expressed as mean \pm SEM and graphed using Prism (GraphPad). One-way ANOVA and Tukey's post-hoc tests were performed to identify statistically significant differences using InStat (GraphPad).

\section{Results}

\section{LRRK2 Interacts with MKK3}

We have recently shown that LRRK2 binds MKK3, -6 and -7. To confirm these results, we cotransfected HEKFT 293 cells with WT LRRK2 \pm MKK3, and immunoprecipitated the proteins. Figure 1 shows that MKK3 is readily detectable by immunoblot following LRRK2 immunoprecipitation.

\section{LRRK2 Interacts with JIP1-4}

The strong association between LRRK2 and MKKsled us to hypothesize that LRRK2 might also interact with the JIPs, and that JIPs might modulate the subcellular translocation of LRRK2 with MKK6. To test this hypothesis, we coexpressed V5His-tagged WT LRRK2 construct with Flag-tagged JIP1-4 constructs in HEK293-FT cells, and performed coimmunoprecipitations to determine whether LRRK2 interacts with the JIPs. Protein complexes containing JIP1-3 (fig. 2a) and JIP4 (fig. 2b) were readily evident upon coimmunoprecipitation of LRRK2 or JIPs. Coexpressing the different proteins also produced a number of additional affects. The expression of LRRK2 
Fig. 1. LRRK2 binds to MKK3. a HEK 293 cells were transfected with V5-LRRK2 (WT) \pm Flag-MKK3. After $24 \mathrm{~h}$, the lysates were collected and V5-LRRK2 immunoprecipitated. The immunoprecipitates were immunoblotted with either anti-V5 antibody (upper panel, to detect LRRK2) or anti-Flag (lower panel, to detect MKK3). b HEK 293 cells were transfected with Flag-MKK3 \pm LRRK2 (WT). After $24 \mathrm{~h}$, the lysates were collected and Flag-tagged MKK3 immunoprecipitated. The immunoprecipitates were immunoblotted with either anti-V5 antibody (upper panel, to detect LRRK2) or anti-Flag (lower panel, to detect MKK3). Arrows point to LRRK2 and MKK3. Representative data from three independent experiments.

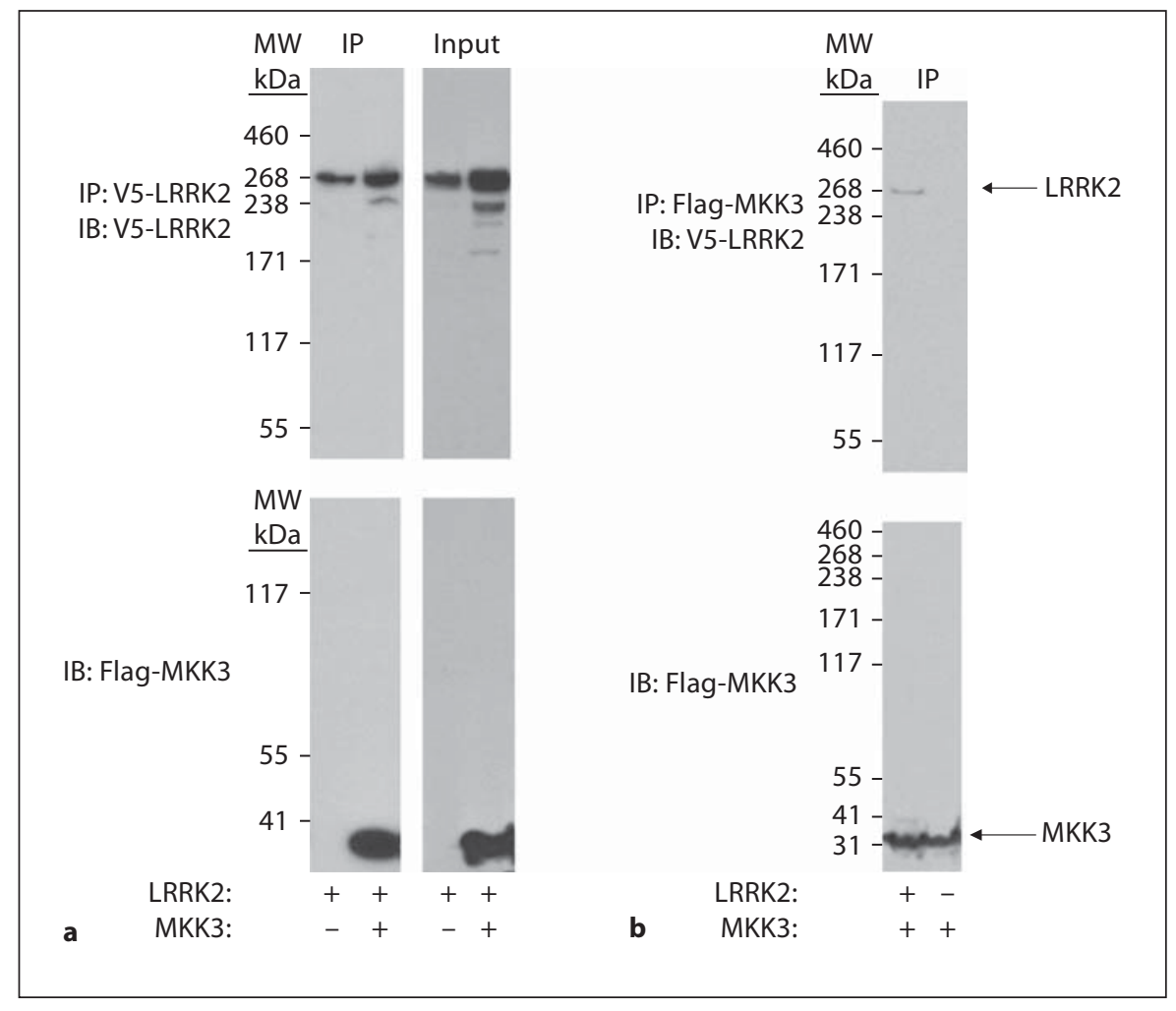

increase formation of high molecular weight bands in the JIP3 and -4 transfected cells (fig. 2). The appearance of high-molecular-weight bands suggests that LRRK2 might stimulate oligomerization of JIP3 and -4. Coexpressing JIP1-4 with LRRK2 also overall increased levels of JIP expression (fig. 2).

\section{LRRK2 Increases Levels of JIPS}

In related work, we showed that the coexpression of MKK6 with LRRK2 induces the expression and translocation of both proteins from the mitochondria to the plasma membrane and the ER, and that this translocation is dependent upon the functionality of MKK6 [27]. We hypothesize that JIPs may be integral components of the complex regulation of the translocation to the plasma membrane. To test this hypothesis, HEK293-FT cells were transfected with or without JIP1-4, and the lysates were fractionated into cytosolic and membrane fractions. The amounts of JIP1-4 were increased in the presence of LRRK2 in both the cytosolic and the membrane fractions (fig. 3). We did not observe that LRRK2 altered the distribution of the JIPs (fig. 3). These results indicate that expressing LRRK2 increases the steady state levels of JIP proteins, but does not alter the gross distribution of JIPs.

\section{LRRK2 Increases JIP4 Ubiquitination}

The ability of LRRK2 to increase levels of JIPs suggests the possibility that LRRK2 modulates degradation of JIPs. To investigate whether LRRK2 modulates the catabolism of JIPs, we investigated whether expressing LRRK2 affects ubiquitination of JIP4. LRRK2 was cotransfected with Flag-tagged JIP4 and HA-tagged ubiquitin. JIP4 was immunoprecipitated and probed with antiHA antibody to determine whether JIP4 ubiquitination is modified by the presence of WT LRRK2 (fig. 4). Coexpressing LRRK2 strongly increased ubiquitination of JIP4 (fig. 4). Immunoblotting for JIP4 also suggested that oligomerization of JIP4 was increased in the presence of LRRK2, although we cannot rule out the possibility that detection of the higher molecular weight species simply reflected elevated levels of JIP4.

\section{JIP4 Oligomerization Is Increased by Disease-Related Mutations in LRRK2}

Our previous studies indicate that G2019S and R1441C mutations in LRRK2 exhibit increased binding with MKK6. This observation suggested the possibility that disease-related LRRK2 mutations also enhance binding to JIPs. To explore the effects of disease-related LRRK2 mu- 
Fig. 2. LRRK2 binds to JIPs. a LRRK2 coimmunoprecipitates with JIP1-3. bLRRK2 coimmunoprecipitates with JIP4. HEK 293 cells were transfected with Flag-tagged JIP1-3 \pm V5-LRRK2 (a) or Flag-tagged JIP4 \pm V5-LRRK2, and the Flag-JIP complex was immunoprecipitated. Specific association of LRRK2 with each JIP is evident in the complexes shown in the immunoblot in the left panel. Representative data from three independent experiments.

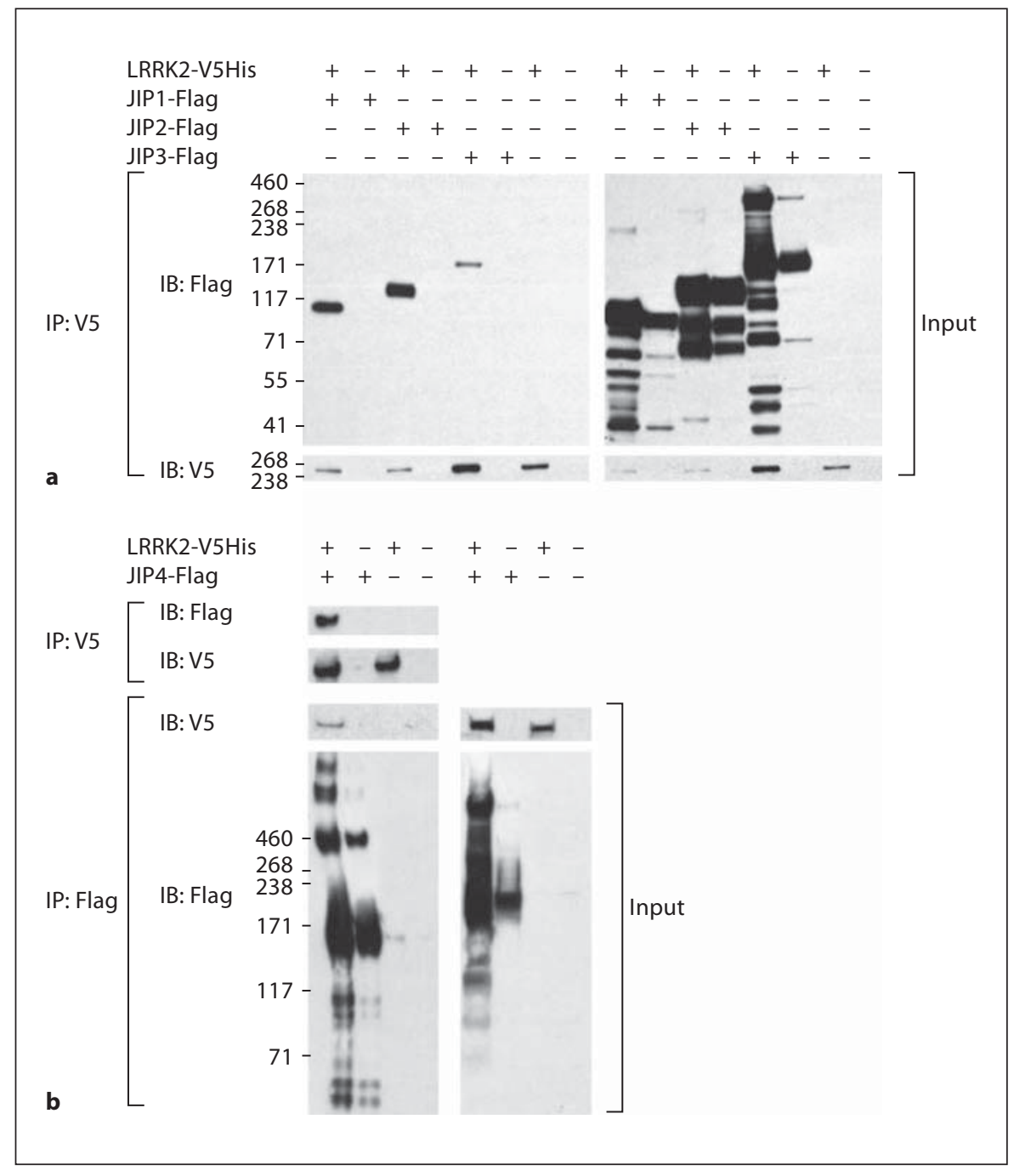

tations on JIP biology, we cotransfected JIP4 with LRRK2 constructs containing mutations linked to disease: G2019S and R1441C, I2020T, Y1699C. The G2019S, R1441C and I2020T mutations were each associated with increased levels of JIP4 and increased levels of oligomerizated complex (fig. 5a). Densitometric quantification indicated that the amount of dimmer was increased for the mutant LRRK2 constructs (fig. 5b), but the apparent increase might also result from the overall increase in total JIP4 levels.

\section{Discussion}

Mutations in LRRK2 are among the most common genetic mutations associated with PD. The mechanism by which mutations might contribute to disease is largely unknown, and indeed, the general biology of LRRK2 is poorly understood. In this paper, we set out to understand one aspect of LRRK2 biology evident from its structure. The kinase domain of LRRK2 is homologous to RIP kinases and mixed lineage kinases, which are classes of kinases known to regulate the stress kinase cascades in part through binding to MKKs. We have now shown that LRRK2 also binds to MKKs. We also demonstrate that LRRK2 interacts with a family of scaffold proteins, termed JIPs, which regulate the stress kinase cascades. Cotransfecting LRRK2 increases JIP expression levels, and interaction of LRRK2 with the JIPs might promote their oligomerization, which is thought to be associated with MAPK activation [28]. Most but not all disease-related mutations in LRRK2 that we studied exhibited high-molecular-weight bands indicative of oligomeriza- 
Fig. 3. LRRK2 increases JIP levels but does not induce translocation. HEK 293 cells were transfected with Flag-tagged JIP1-3 \pm V5-LRRK2 or Flag-tagged JIP4 \pm V5LRRK2. The resulting lysates were separated into cytoplasmic (upper panel) or membrane (lower panel) components. Cotransfecting LRRK2 increased JIP levels in both the cytoplasmic and membrane fractions equally. Representative data from three independent experiments.

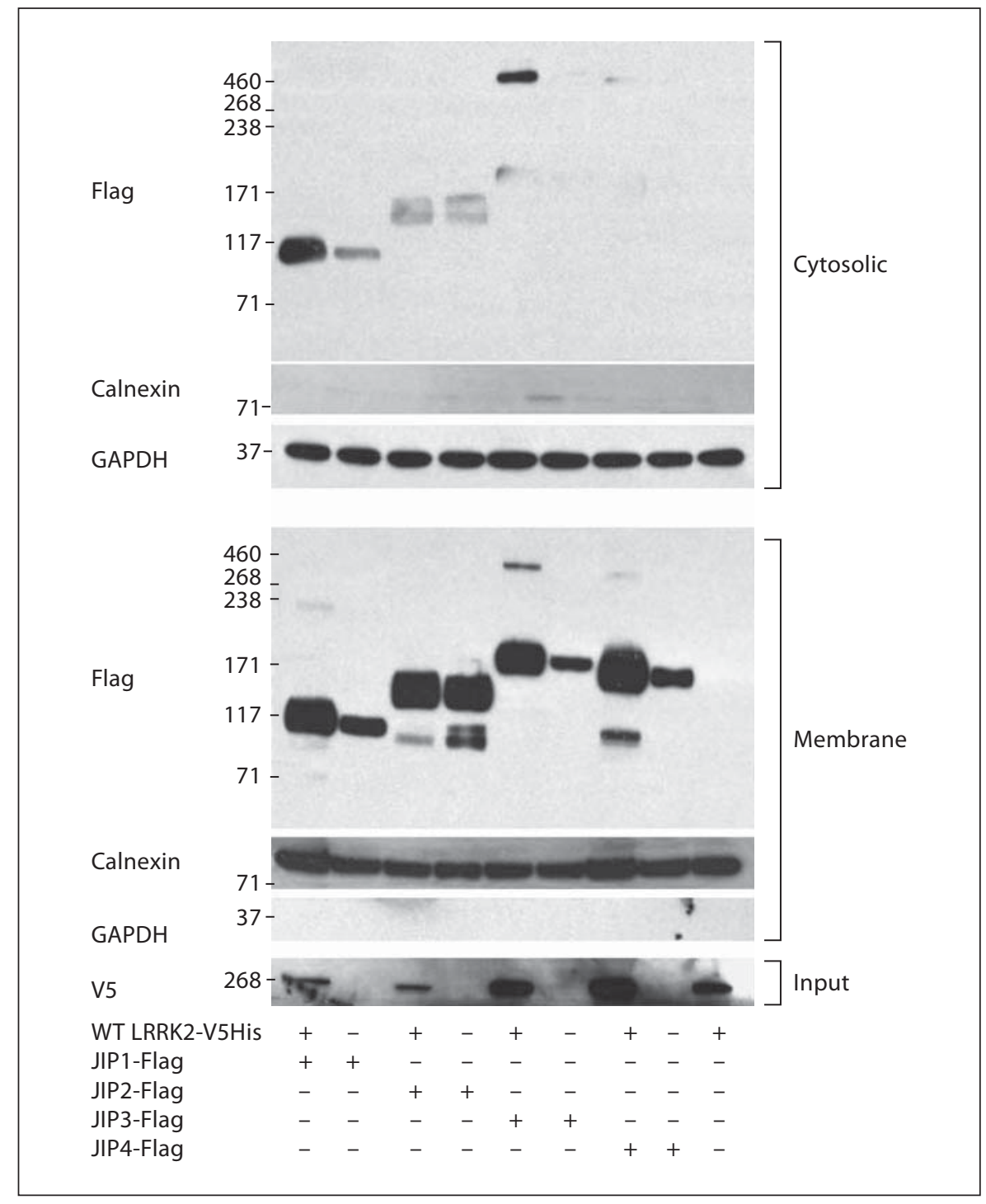

tion. Interestingly, cotransfecting LRRK2 with JIP4, and possibly other JIPs, also increases levels of ubiquitinated JIP4, although the significance of this change is unclear.

Our observation that LRRK2 associates with MKKs and with JIPs is consistent with an increasing body of evidence suggesting that LRRK2 regulates the stress kinase cascade. Studies in Caenorhabditis elegans demonstrate that LRRK2 regulates the response to mitochondrial stress (complex I inhibition), and that the response requires the activity of the nematode homologues of MKK6 and p38 [27, 33]. LRRK2 is known to autophosphorylate, although the amount of autophosphorylation is very low. Gloeckner et al. [26] recently demonstrated that LRRK2 can transphosphorylate MKK 3-7 in vitro.
The reaction does not appear to be catalytic, though, because levels of MKK phosphorylation were similar to levels of LRRK2 autophosphorylation, despite addition of more than a 10,000-fold excess of MKK. Phosphorylation of MKK by LRRK2 occurs in the amino-domain, which is far from the sites normally associated with MKK activation [26]. The low level of phosphorylation is consistent with a model in which LRRK2 binds the MKKs, phosphorylates the proteins, but then either does not release the MKK or becomes catalytically inactive. Studies from our laboratory demonstrate that LRRK2 binds to MKKs, but interestingly, we find evidence for phosphorylation of MKKs by LRRK2 in vitro, but not in vivo [27]. Our results suggest that binding of LRRK2 to MKK2 increases the 


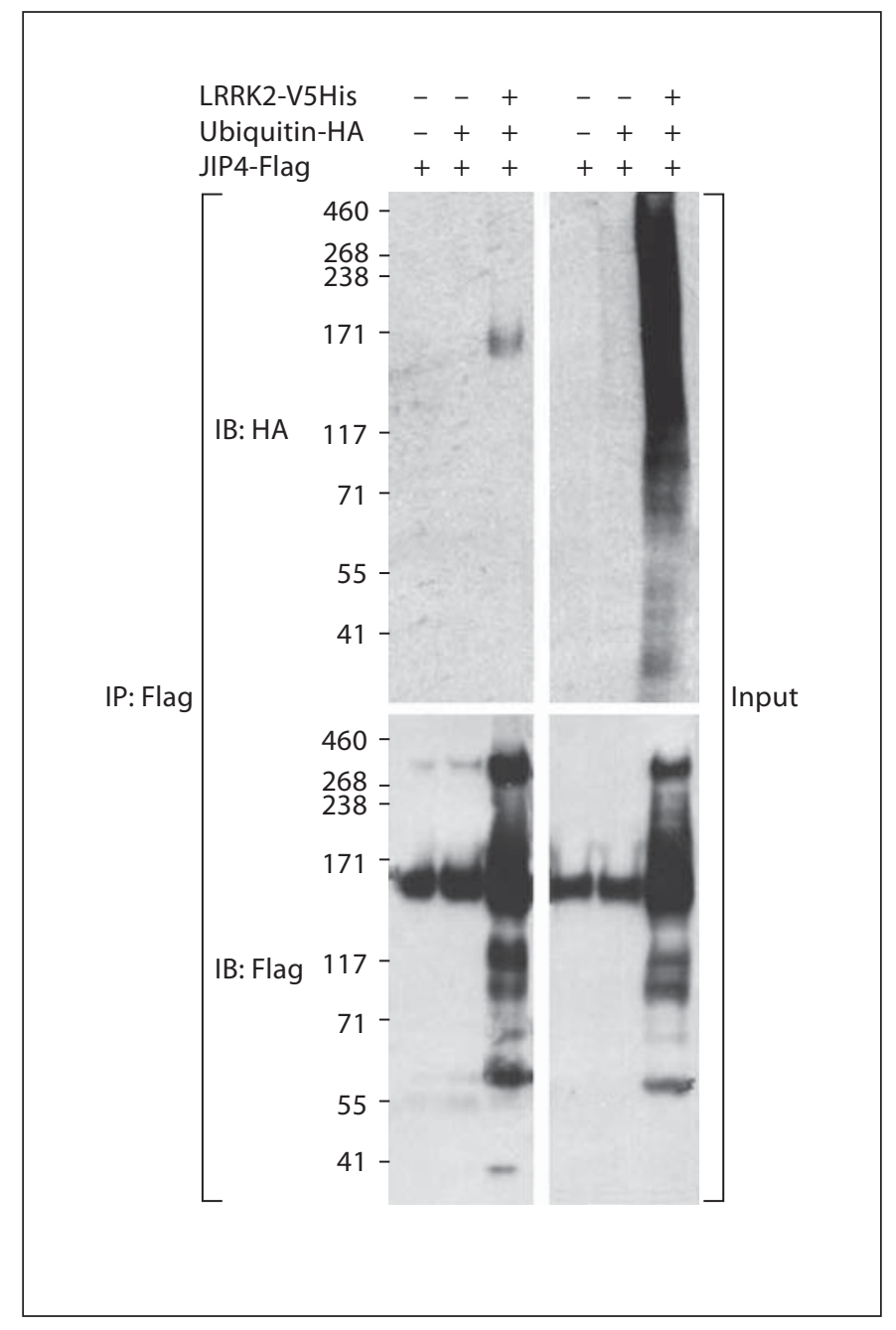

Fig. 4. LRRK2 increases JIP ubiquitination. HEK 293 cells were transfected with Flag-tagged JIP1-3, V5-LRRK2 \pm HA ubiquitin. The lysates were subjected to immunoprecipitation with antiFlag antibody, and probed with antibodies to V5 (for LRRK2), HA (for ubiquitin) or Flag (for JIP4). Cotransfection increased oligomerization of JIP4, and resulted in immunoprecipitation (IP) of ubiquitinated JIP4. Representative data from three independent experiments.

expression and membrane localization of both proteins, as shown by immunocytochemistry and biochemical fractionation.

The interaction of LRRK2 with JIPs is consistent with the observed interaction between LRRK2 and MKKs [27]. JIPs are scaffold proteins for the stress kinase cascade [28]. The ability of LRRK2 to bind JIPs in absence of cotransfected MKKs suggests that LRRK2 binds JIPs directly, or at least through a mechanism that does not require MKKs. Thus, the JIP scaffolding complex appears

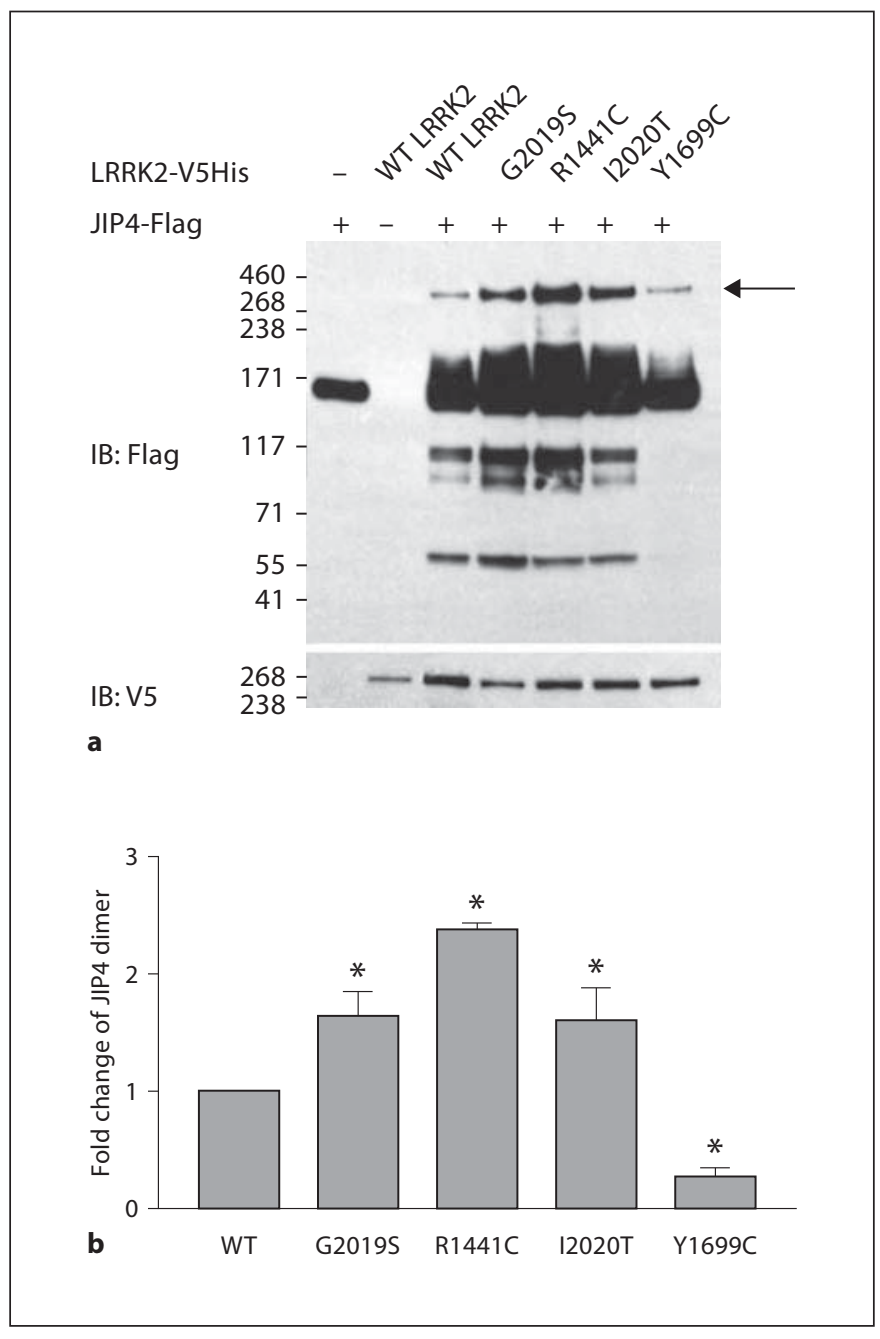

Fig. 5. Disease-linked mutations in LRRK2 increase JIP oligomerization. HEK 293 cells were transfected with Flag-tagged JIP1-3, V5-LRRK2 (WT, G2019S, R1441C, I2020T or Y1699C) \pm HA ubiquitin. JIP4 was immunoprecipitated (a) and the amount of oligomeric JIP4 quantified (b). a The arrow points to the oligomer bands used for quantification, and quantification was relative to total JIP4 levels. $\mathbf{b} * \mathrm{p}<0.05(\mathrm{n}=3)$. function to bring together MKKs with MAPKs (e.g. JNK and p38), and also to direct binding to different sites within the cells. Some JIPs tend to direct binding towards the membrane, such as JIP4, while JIP1-3 are known to bind to microtubules. The interaction of LRRK2 with JIP1-3 suggests that LRRK2 might bind to JIP1-3 as a cargo protein, and that binding might facilitate transport along microtubules. Such transport might be particularly important in neurons where LRRK2 can be found in 
synaptic fields, such as in the striatum. Other proteins linked to disease also appear to interact with JIPs. $\alpha$ Synuclein was recently shown to regulate levels of JIP1 [34]. Amyloid precursor protein binds to JIP1 in much the same manner, although the biology of the interaction remains unclear [35].

LRRK2 exhibits a striking ability to increase total levels of JIPs and ubiquitination of JIPs. These two effects in combination suggest that LRRK2 inhibits degradation of JIPs, either through the proteasomal or autophagic systems. Alternatively, LRRK2 might also act to increase JIP translation or transcription. A link between LRRK2 and regulation of protein translation is suggested by the observation that LRRK2 binds and phosphorylates 4E-BP, a regulator of protein translation machinery [24]. A putative role in regulating protein degradation is consistent with the presence of ubiquitin-positive inclusions in most cases of PD that are associated with LRRK2 mutations [5]. Most of these inclusions contain $\alpha$-synuclein, but some inclusions contain tau protein $[5,36]$. The putative ability of LRRK2 to inhibit autophagy or proteasomal function could contribute to increased risk of PD and inclusion formation among subjects with LRRK2 mutations. Several reports also note that LRRK2 overexpression leads to the formation of inclusions containing LRRK2 [20,37]. Other reports indicate that LRRK2 regulates autophagy [38]. Inhibition of autophagy or proteasomal function could induce inclusion, stimulate inclusion formation as well as increase levels of JIP and JIP oligomers. Further experiments are needed to determine the precise mechanism.

In summary, our data suggest that a strong biological connection exists between LRRK2, the stress kinase cascade and JIPs, a class of scaffold proteins that bind stress kinases. The role of stress kinases in cell death creates a cogent linkage between LRRK2 and cell death. Whether this pathway is the actual pathway linked to the pathophysiology of disease among subjects carrying mutations in LRRK2 remains to be determined. Most of the mutant LRRK2 constructs increased JIP4 levels and oligomerization more than WT with the exception of Y1699C. The incomplete correlation between pathological LRRK2 mutations and enhanced JIP expression/oligomerization suggests that this axis is not the principle mechanism by which LRRK2 causes disease. However, understanding the mechanisms by which LRRK2 modulates the stress kinase cascade could lead to increased understanding of the biology of LRRK2 and potential differences in the pathophysiology of disease among individuals with different LRRK2 mutations.

\section{Acknowledgements}

We thank Roger Davis for his generosity in sharing the JIP plasmids. This work was supported by grant awards to B.W. from the Alzheimer Association, the Michael J. Fox Foundation, NIEHS ES15567, and NINDS NS060872.

\section{References}

1 Biskup S, Gerlach M, Kupsch A, Reichmann H, Riederer P, Vieregge P, Wullner U, Gasser T: Genes associated with Parkinson syndrome. J Neurol 2008;255(suppl 5):8-17.

-2 Taylor JP, Mata IF, Farrer MJ: LRRK2: a common pathway for parkinsonism, pathogenesis and prevention? Trends Mol Med 2006; 12:76-82.

-3 Ozelius LJ, Senthil G, Saunders-Pullman R, Ohmann E, Deligtisch A, Tagliati M, Hunt AL, Klein C, Henick B, Hailpern SM, Lipton RB, Soto-Valencia J, Risch N, Bressman SB: LRRK2 G2019S as a cause of Parkinson's disease in Ashkenazi Jews. N Engl J Med 2006; 354:424-425.

-4 Lesage S, Durr A, Tazir M, Lohmann E, Leutenegger AL, Janin S, Pollak P, Brice A: LRRK2 G2019S as a cause of Parkinson's disease in North African Arabs. N Engl J Med 2006;354:422-423.
5 Zimprich A, Biskup S, Leitner P, Lichtner P, Farrer M, Lincoln S, Kachergus J, Hulihan M, Uitti RJ, Calne DB, Stoessl AJ, Pfeiffer RF, Patenge N, Carbajal IC, Vieregge P, Asmus F, Muller-Myhsok B, Dickson DW, Meitinger T, Strom TM, Wszolek ZK, Gasser T: Mutations in LRRK2 cause autosomal-dominant parkinsonism with pleomorphic pathology. Neuron 2004;44:601-607.

-6 Paisan-Ruiz C, Jain S, Evans EW, Gilks WP, Simon J, van der Brug M, Lopez de Munain A, Aparicio S, Gil AM, Khan N, Johnson J, Martinez JR, Nicholl D, Carrera IM, Pena AS, de Silva R, Lees A, Marti-Masso JF, Perez-Tur J, Wood NW, Singleton AB: Cloning of the gene containing mutations that cause PARK8-linked Parkinson's disease. Neuron 2004;44:595-600.

-7 Festjens N, Vanden Berghe T, Cornelis S, Vandenabeele P: RIP1, a kinase on the crossroads of a cell's decision to live or die. Cell Death Differ 2007;14:400-410.
8 Meylan E, Tschopp J: The RIP kinases: crucial integrators of cellular stress. Trends Biochem Sci 2005;30:151-159.

9 Ho CC, Rideout HJ, Ribe E, Troy CM, Dauer WT: The Parkinson disease protein leucinerich repeat kinase 2 transduces death signals via Fas-associated protein with death domain and caspase- 8 in a cellular model of neurodegeneration. J Neurosci 2009;29:1011-1016.

10 Biskup S, Moore DJ, Celsi F, Higashi S, West AB, Andrabi SA, Kurkinen K, Yu SW, Savitt JM, Waldvogel HJ, Faull RL, Emson PC, Torp R, Ottersen OP, Dawson TM, Dawson VL: Localization of LRRK2 to membranous and vesicular structures in mammalian brain. Ann Neurol 2006;60:557-569.

11 West AB, Moore DJ, Choi C, Andrabi SA, Li X, Dikeman D, Biskup S, Zhang Z, Lim KL, Dawson VL, Dawson TM: Parkinson's disease-associated mutations in LRRK2 link enhanced GTP-binding and kinase activities to neuronal toxicity. Hum Mol Genet 2007;16:223-232. 
-12 Lewis PA, Greggio E, Beilina A, Jain S, Baker A, Cookson MR: The R1441C mutation of LRRK2 disrupts GTP hydrolysis. Biochem Biophys Res Commun 2007;357:668-671.

13 Deng J, Lewis PA, Greggio E, Sluch E, Beilina A, Cookson MR: Structure of the ROC domain from the Parkinson's disease-associated leucine-rich repeat kinase 2 reveals a dimeric GTPase. Proc Natl Acad Sci USA 2008;105:1499-1504.

14 Greggio E, Zambrano I, Kaganovich A, Beilina A, Taymans JM, Daniels V, Lewis P, Jain S, Ding J, Syed A, Thomas KJ, Baekelandt V, Cookson MR: The Parkinson disease-associated leucine-rich repeat kinase 2 (LRRK2) is a dimer that undergoes intramolecular autophosphorylation. J Biol Chem 2008;283: 16906-16914.

-15 Klein CL, Rovelli G, Springer W, Schall C, Gasser T, Kahle PJ: Homo- and heterodimerization of ROCO kinases: LRRK2 kinase inhibition by the LRRK2 ROCO fragment. J Neurochem 2009;111:703-715

$\checkmark 16$ Sen S, Webber PJ, West AB: Dependence of leucine-rich repeat kinase 2 (LRRK2) kinase activity on dimerization. J Biol Chem 2009; 284:36346-36356.

17 Gotthardt K, Weyand M, Kortholt A, Van Haastert PJ, Wittinghofer A: Structure of the Roc-COR domain tandem of C. tepidum, a prokaryotic homologue of the human LRRK2 Parkinson kinase. EMBO J 2008;27: 2239-2249.

-18 Wang LH, Besirli CG, Johnson EM Jr: Mixedlineage kinases: a target for the prevention of neurodegeneration. Annu Rev Pharmacol Toxicol 2004;44:451-474.

19 West AB, Moore DJ, Biskup S, Bugayenko A, Smith WW, Ross CA, Dawson VL, Dawson TM: Parkinson's disease-associated mutations in leucine-rich repeat kinase 2 augment kinase activity. Proc Natl Acad Sci USA 2005;102:16842-16847.

-20 Greggio E, Jain S, Kingsbury A, Bandopadhyay R, Lewis P, Kaganovich A, van der Brug MP, Beilina A, Blackinton J, Thomas KJ, Ahmad R, Miller DW, Kesavapany S, Singleton A, Lees A, Harvey RJ, Harvey K, Cookson MR: Kinase activity is required for the toxic effects of mutant LRRK2/dardarin. Neurobiol Dis 2006;23:329-341.
21 Greggio E, Lewis PA, van der Brug MP, Ahmad R, Kaganovich A, Ding J, Beilina A, Baker AK, Cookson MR: Mutations in LRRK2/dardarin associated with Parkinson disease are more toxic than equivalent mutations in the homologous kinase LRRK1. J Neurochem 2007;102:93-102.

22 Biskup S, West AB: Zeroing in on LRRK2 linked pathogenic mechanisms in Parkinson's disease. Biochim Biophys Acta 2009; 1792:625-633.

23 Greggio E, Cookson MR: Leucine-rich repeat kinase 2 mutations and Parkinson's disease: three questions. ASN Neuro 2009;1: e00002.

24 Imai Y, Gehrke S, Wang HQ, Takahashi R, Hasegawa K, Oota E, Lu B: Phosphorylation of $4 \mathrm{E}-\mathrm{BP}$ by LRRK2 affects the maintenance of dopaminergic neurons in Drosophila. EMBO J 2008;27:2432-2443.

25 Jaleel M, Nichols RJ, Deak M, Campbell DG, Gillardon F, Knebel A, Alessi DR: LRRK2 phosphorylates moesin at threonine-558: characterization of how Parkinson's disease mutants affect kinase activity. Biochem J 2007;405:307-317.

26 Gloeckner CJ, Schumacher A, Boldt K, Ueffing $\mathrm{M}$ : The Parkinson disease-associated protein kinase LRRK2 exhibits MAPKKK activity and phosphorylates MKK3/6 and MKK4/7, in vitro. J Neurochem 2009;109: 959-968.

27 Hsu CH, Chan D, Greggio E, Guillily MD, Segal L, Saha S, Cookson MR, Wolozin B: Association of Parkinson's disease-related protein LRRK2 with MKK6 stimulates their mutual membrane translocation. J Neurochem 2010; Jan 7, Epub ahead of print.

28 Whitmarsh AJ: The JIP family of MAPK scaffold proteins. Biochem Soc Trans 2006; 34:828-832

29 Kelkar N, Standen CL, Davis RJ: Role of the JIP4 scaffold protein in the regulation of $\mathrm{mi}$ togen-activated protein kinase signaling pathways. Mol Cell Biol 2005;25:2733-2743.
30 Verhey KJ, Meyer D, Deehan R, Blenis J, Schnapp BJ, Rapoport TA, Margolis B: Cargo of kinesin identified as JIP scaffolding proteins and associated signaling molecules. J Cell Biol 2001;152:959-970.

-31 Zerbinatti CV, Cordy JM, Chen CD, Guillily M, Suon S, Ray WJ, Seabrook GR, Abraham CR, Wolozin B: Oxysterol-binding protein-1 (OSBP1) modulates processing and trafficking of the amyloid precursor protein. Mol Neurodegener 2008;3:5.

32 Ostrerova N, Petrucelli L, Farrer M, Mehta N, Alexander P, Choi P, Palacino J, Hardy J, Wolozin B: $\alpha$-Synuclein shares physical and functional homology with 14-3-3 proteins. J Neurosci 1999;19:5782-5791.

-33 Saha S, Guillily MD, Ferree A, Lanceta J, Chan D, Ghosh J, Hsu CH, Segal L, Raghavan K, Matsumoto K, Hisamoto N, Kuwahara T, Iwatsubo T, Moore L, Goldstein L, Cookson $\mathrm{M}$, Wolozin B: LRRK2 modulates vulnerability to mitochondrial dysfunction in Caenorhabditis elegans. J Neurosci 2009;29: 9210-9218.

34 Hashimoto M, Hsu LJ, Rockenstein E, Takenouchi T, Mallory M, Masliah E: alphaSynuclein protects against oxidative stress via inactivation of the c-Jun $\mathrm{N}$-terminal kinase stress-signaling pathway in neuronal cells. J Biol Chem 2002;277:11465-11472.

35 Scheinfeld MH, Ghersi E, Davies P, D’Adamio L: Amyloid beta protein precursor is phosphorylated by JNK-1 independent of, yet facilitated by, JNK-interacting protein (JIP)-1. J Biol Chem 2003;278:42058-42063.

-36 Rajput A, Dickson DW, Robinson CA, Ross OA, Dachsel JC, Lincoln SJ, Cobb SA, Rajput ML, Farrer MJ: Parkinsonism, Lrrk2 G2019S, and tau neuropathology. Neurology 2006;67: 1506-1508.

37 Smith WW, Pei Z, Jiang H, Moore DJ, Liang Y, West AB, Dawson VL, Dawson TM, Ross CA: Leucine-rich repeat kinase 2 (LRRK2) interacts with parkin and mutant LRRK2 induces neuronal degeneration. Proc Natl Acad Sci USA 2005;102:18676-18681.

$>38$ Plowey ED, Cherra SJ 3rd, Liu YJ, Chu CT: Role of autophagy in G2019S-LRRK2-associated neurite shortening in differentiated SH-SY5Y cells. J Neurochem 2008;105:10481056. 